\title{
A Downlink Max-SINR Precoding for Massive MIMO System
}

\author{
Jiang Jing and $\mathrm{Xu}$ Zheng \\ Xi'an University of Posts \& Telecommunications, Xi'an, China \\ Jiangjing18@gmail.com; xuzheng0524@163.com
}

\begin{abstract}
To acquire the maximal array gain and mitigate the impact of pilot contamination, the downlink precoding algorithm on Max-SINR criterion was investigated and improved for Massive MIMO system. The objective function assured to maximize the utilization rate of the transmission power under the condition that SINR is not lower than the desired threshold. The Lagrangian function was deduced according to the objective function. Further SINR computation counted the channel estimation error caused by pilot contamination and its computational complexity was reduced by utilizing the character of Massive MIMO channel. At last the optimal downlink precoding matrix was obtained by KKT conditions. When base station configured 100 antennas and the pilot contamination was severe, numerical results proved that the spectrum efficiency of the proposed algorithm was 1.9 times higher than the popular Massive MIMO precoding --- MF algorithm. The proposed algorithm can effectively mitigate the impact of pilot contamination and achieve high throughput with maximizing gain, and is valuable to Massive MIMO system.
\end{abstract}

Keywords: Massive MIMO, Large scale MIMO, Pilot contamination, Precoding, $\operatorname{Max}-$ SINR

\section{Introduction}

Massive MIMO is an emerging technology which scales up MIMO to hundreds or even thousands antennas $[1,2]$. Both the spectral efficiency and cell capacity will be greatly improved by enormous array gains and interference suppression gains. The researches on Massive MIMO focus on the following developments: the channel capacity [1-6], precoding technology [7-14], MIMO channel training [15-17] and Massive MIMO channel modeling [15-17].

The main objective of precoding in Massive MIMO system is to improve the gain of large-scale antenna array and mitigate the impact of pilot contamination. Linear precoding, e.g. Match Filter algorithm, can achieve the optimal performance because noise can be averaged by massive antenna, on the other hand the interference from other cells isn't serious anymore for precisely focused beams. Literature [2-8] analyzed and evaluated the upper bound of the performance on popular Massive MIMO precoding algorithms, e.g. matched-filter (MF), zero forcing (ZF), dirty paper coding (DPC), and proved that the performance of Massive MIMO was limited by the pilot contamination. According the channel reciprocity, the downlink precoding matrix was calculated on uplink measured channel through the pilot training. Orthogonal pilot training sequences in the coherence time are finite, so non-orthogonal pilot training sequences may be reused in adjacent cells. The estimated channel state information would be polluted by non-orthogonal uplink pilots and it is called Pilot Contamination [1-2].

To improve the gain of large scale antenna array, literature [9-11] improved traditional MIMO or Beamforming algorithms according to the characters of Massive MIMO. Literature [9] proposed Single-layer Beamforming (BF) and multi-layer BF. The array gains were the product of direction gains and the array efficiency of the large-scale 
MIMO for Single-layer BF. The weighting vector of Single-layer BF was the direction whose array gain was maximal. For the multi-layer BF, the optimal BF matrixes that maximized the user's sum-rate was a compromise between the multiplexing gain and the inter-beam interference. Literature [10] presented the successive power allocation method with Minimum Mean Square Error (MMSE) filters. For the large-scale random matrices, the empirical eigenvalue distributions converged to an asymptotic limit. The proposed algorithm utilized the limit and other finite parameters to approximate the sum rate. Literature [11] designed precoders maximizing the ergodic mutual information (EMI) equipped with MMSE filters. To optimize the EMI approximation, it was deduced that the relative error between the approximation and its actual value was $o\left(1 / t^{2}\right.$, term (t is the number of the transmitting antenna).

Literature [12-14] designed precoders to reduce the pilot contamination. Literature [12] put forward a multi-cell MMSE (Minimum Mean Square Error) algorithm whose MMSE contain two parts: the Mean Square error between the decoding signals and transmitting signals, and the interference power of other cells. The precoding matrix was deduced on MMSE criterion. Literature [13] proved that it could reduce inter-cell interference and obtain better channel estimates through shifting the location of pilots in time frames of neighboring cells. Literature [14] proposed a pilot contamination precoding which is the product of popular precoding matrixes and slow-fading coefficients matrix from the target user to the base station of the local cell to other cells.

Max-SINR (Maximize Signal-to-Interference-plus-Noise Ratio) had been an important criterion in the research of interference cancelling and used in Multi-user MIMO precoding and cooperative transmission of Multi-cells [18-25]. Literature [18] proposed Max-SINR algorithm of multi-cell cooperative beamforming. To reduce the complication, it utilized statistical channel information of beamformers and two iterations to achieve Max-SINR. Literature [19] investigated two objective functions: Quality-of-Service (QoS) and Max-Min SINR (MMS) criterion under per-base station's power constraints, and then proposed an efficient algorithm to solve the MMS problem in an approximate manner. Literature [21] proposed max-min SINR beamforming between the virtual uplink and the multi-cell downlink considering per-BS power constraints. The virtual uplink optimization was solved by a hierarchical iterative scheme, and then converted to achieve the solution to the multi-cell downlink beamforming. It achieved better performance in terms of both the worst-user rate and the rate per energy.

In this paper, Max SINR will be investigated and improved for Massive MIMO precoding. The interference part of SINR counts the impact of the channel estimation error and the character of MIMO channel. The objective function assures to maximize the utilization rate of the transmission power under the condition that SINR is not lower than the desired threshold. Then we optimize the Lagrangian function by KKT conditions and obtain the optimal downlink precoding matrix. Numerical results proved that the proposed algorithm can effectively mitigate the impact of pilot contamination and achieve high throughput with maximizing the gains of transmitting power. 


\section{System Model}

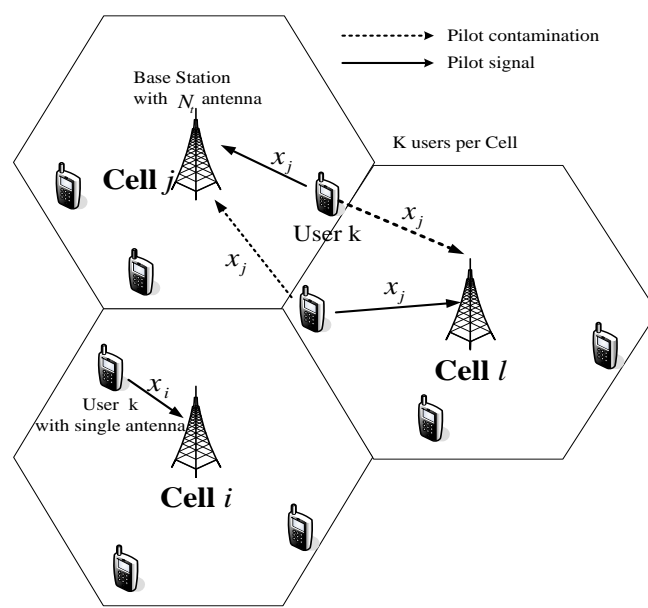

Figure 1. Uplink System Model of Massive MIMO

There are $I$ cells and each cell serves $K$ User Equipments (UE) in Massive MIMO system shown as Figure 1. Base station of each cell is equipped with $N_{t}$ antenna and per user equipment configures a single antenna. In the uplink training, the receiving signal of the base station in the cell $j$ is denoted as flowing:

$$
\boldsymbol{y}_{j}=\sum_{l=1}^{L} \sum_{k=1}^{K} \sqrt{p_{r} \tau \beta_{l k}^{j}} \boldsymbol{h}_{l k}^{j} \boldsymbol{x}_{j}+\sum_{i=1, i \neq l}^{I} \sum_{k=1}^{K} \sqrt{p_{r} \tau \beta_{i k}{ }_{i k}^{j}} \boldsymbol{h}_{i k}^{j} \boldsymbol{x}_{i}+\boldsymbol{n}_{j}
$$

Where $\boldsymbol{x}_{j}$ are pilot signals reused by $L$ cells, $\boldsymbol{x}_{i}$ are pilot signals used by cell $i$ whose pilot sequences is orthogonal with $x_{j}$, and the sequence length of uplink pilots is $\tau$ and $x_{i} x_{j}^{H}=\delta_{i j} \cdot \beta_{l k}^{j}$ are real slow coefficients from $k$ UEs of cell $l$ to Base Station of cell $j . \boldsymbol{h}_{l k}^{j}$ is the fast fading matrix from $k$ UEs of cell $l$ to Base Station of cell $j$ and each element is independent and identically distributed (i.i.d.) with $C N(0,1)$. $p_{r}$ is the average power (during transmission) of each user. So the first part of (1) is the received signal of reused pilots and the second part of (1) is the received signal of orthogonal pilot signals.

LS algorithm is applied for the channel estimation of the uplink training. The estimated channel state information $\hat{\boldsymbol{H}}_{j k}^{j}$ from k UEs of cell $j$ to Base Station of cell $j$ :

$$
\hat{\boldsymbol{H}}_{j k}^{j}=\boldsymbol{y}_{j} \cdot \boldsymbol{x}_{j}^{H} / \sqrt{p_{r} \tau}=\sqrt{\beta_{j k}^{j}} \boldsymbol{h}_{j k}^{j}+\sum_{l=1, l \neq j}^{L} \sum_{k=1}^{K} \sqrt{\beta_{l k}^{j}} \boldsymbol{h}_{l k}^{j}+\boldsymbol{n}_{j}^{\prime}
$$

Where the first section $\sqrt{\beta_{j k}^{j}} h_{j k}^{j}$ is the true channel information and the second section $\sum_{l=1, l \neq j}^{L} \sum_{k=1}^{K} \sqrt{\beta_{l k}^{j}} \boldsymbol{h}_{l k}^{j}$ denotes the pilot contamination from reused pilots. The noise term is $\boldsymbol{n}_{j}^{\prime}=\frac{\boldsymbol{n}_{j} \boldsymbol{x}_{j}^{H}}{\sqrt{p_{r} \tau}} \quad \boldsymbol{n}_{j}^{\prime} \in C N\left(0, \frac{\boldsymbol{I}_{\boldsymbol{N}_{t} \times N_{t}}}{\sqrt{p_{r} \tau}}\right)$. If the downlink precoding directly applies the estimated channel state information $\hat{\boldsymbol{H}}_{j k}^{j}$ to calculate the precoding matrix, the accuracy of the downlink precoding must be influenced by the pilot contamination. Finally the performance gains of large-scale array would be greatly degraded for the inaccurate 
precoding.

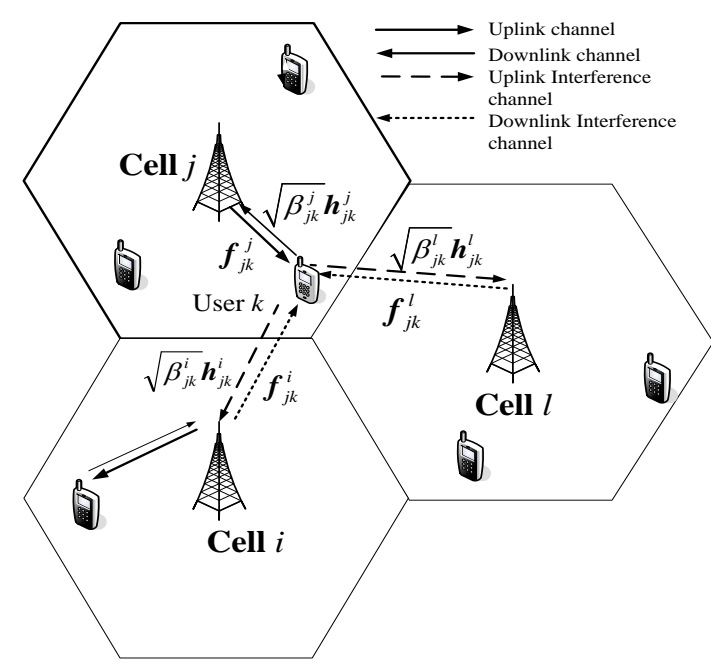

Figure 2. Downlink System Model of Massive MIMO

For the downlink, the receiving signal of user $k$ in cell $j$ is expressed as following:

$$
\boldsymbol{y}_{j k}=\sqrt{p_{f}} \boldsymbol{f}_{j k}^{j} \boldsymbol{a}_{j k} \boldsymbol{q}_{j k}+\sum_{i=1, i \neq j}^{I} \sum_{k=1}^{K} \sqrt{p_{f}} \boldsymbol{f}_{j k}^{i} \boldsymbol{a}_{i k} \boldsymbol{q}_{i k}+z_{j k}
$$

Where $p_{f}$ is the transmitting power of the base station. $\boldsymbol{q}_{i k}$ is downlink transmission signals and $E\left[\boldsymbol{q}_{i k} \boldsymbol{q}_{i k}^{H}\right]=\boldsymbol{I}, \boldsymbol{a}_{j k}$ is the precoding matrix and $\operatorname{tr}\left\{\boldsymbol{a}_{i k}^{H} \boldsymbol{a}_{i k}\right\}=1 . \boldsymbol{f}_{j k}^{j}$ is the channel matrix from the base station of cell $j$ to $\mathrm{UE} k$ of cell $j$ and $f_{j k}^{j}=\sqrt{\beta_{j k}^{j}} h_{j k}^{j^{T}}$ according to the channel reciprocity shown in Figure 2, and $(.)^{T}$ denotes the transposition of the matrix. $f_{j k}^{i}$ is the channel matrix from the base station of cell $i$ to $\mathrm{UE} k$ of cell $j \cdot z_{j k}$ is the add Gaussian noise of $\mathrm{UE} k$ of cell $j$ and i.i.d. random variables with $C N(0,1)$. In (3), the first section is the desired signal and the second part is the interference from adjacent cells.

\section{Downlink Precoding Algorithm Based on MAX-SINR Criterion}

According to (3), downlink SINR of user $k$ in cell $j$ is expressed as following:

$$
\operatorname{SINR}_{j k}=\frac{\left|f_{j k}^{j} \boldsymbol{a}_{j k}\right|^{2}}{1+\sum_{i=1, i \neq j}^{I} \sum_{k=1}^{K}\left|f_{j k}^{i} \boldsymbol{a}_{i k}\right|^{2}}
$$

According to the channel reciprocity, the downlink channel is equal to the uplink channel and $\boldsymbol{f}_{j k}^{j}=\boldsymbol{H}_{j k}^{j^{T}}$. We define the real channel information $\boldsymbol{H}_{j k}^{j}=\sqrt{\beta_{j k}^{j}} \boldsymbol{h}_{j k}^{j}$. It can't be acquired in solving the downlink precoding matrix and is usually replaced by the estimated channel information $\hat{\boldsymbol{H}}_{j k}^{j}$. In the previous analysis, the estimated channel state information contains the real channel information and the pilot pollution from cells using non-orthogonal pilot as denoted in (2). To consider the impact of pilot contamination, the real channel information can be rewritten as following: 


$$
\boldsymbol{H}_{j k}^{j}=\hat{\boldsymbol{H}}_{j k}^{j}-\sum_{l=1, l \neq j}^{L} \sum_{k=1}^{K} \sqrt{\beta_{l k}^{j}} \boldsymbol{h}_{l k}^{j}-n
$$

After the uplink channel is replaced by the real downlink channel, the power of desired signals is calculated as following:

$$
\begin{aligned}
& \left|\boldsymbol{f}_{j k}^{j} \boldsymbol{a}_{j k}\right|^{2}=\left|\boldsymbol{H}_{j k}^{j^{T}} \boldsymbol{a}_{j k}\right|^{2}=\left|\left(\hat{\boldsymbol{H}}_{j k}^{j}-\sum_{l=1, l \neq j}^{L} \sum_{k=1}^{K} \sqrt{\beta_{l k}^{j}} \boldsymbol{h}_{l k}^{j}-\boldsymbol{n}\right)^{T} \boldsymbol{a}_{j k}\right|^{2}= \\
& \left|\hat{\boldsymbol{H}}_{j k}^{{ }^{j}{ }^{T}} \boldsymbol{a}_{j k}\right|^{2}+\left|\left(\sum_{l=1, l \neq j}^{L} \sum_{k=1}^{K} \sqrt{\beta_{l k}^{j}} \boldsymbol{h}_{l k}^{j}\right)^{T} \boldsymbol{a}_{j k}\right|^{2}+\frac{\boldsymbol{I}}{\sqrt{p_{r} \tau}}
\end{aligned}
$$

Because the channel from user $k$ of cell $l$ to the base station of cell $j$ is independent with the channel estimation $\hat{\boldsymbol{H}}_{j k}^{j}$ and the channel is independent with noise. The Cross-product term in (6) is equal to zero. Where $\left|\hat{\boldsymbol{H}}_{j k}^{{ }^{j}{ }^{T}} a_{j k}\right|^{2}$ is the power of desired signals, $\left|\left(\sum_{l=1, l \neq j}^{L} \sum_{k=1}^{K} \sqrt{\beta_{l k}^{j}} h_{l k}^{j}\right)^{T} a_{j k}\right|^{2}$ is the pollution power in the downlink precoding caused by uplink pilot contamination. To maximize $S_{S I N R}{ }_{j k}$, the precoding matrix should not only achieve the maximal gain of desired signals, but also minimize the power of pilot pollution and the interference power. So SINR in (3) is rewritten as following:

$$
\operatorname{SINR}_{j k}=\frac{\left|\sqrt{\beta_{j k}^{j}} \hat{\boldsymbol{H}}_{j k}^{j^{T}} \boldsymbol{a}_{j k}\right|^{2}}{1+\left|\sum_{l=1, l \neq j}^{L} \sum_{k=1}^{K} \sqrt{\beta_{l k}^{j}} \boldsymbol{h}_{l k}^{j^{T}} \boldsymbol{a}_{j k}\right|^{2}+\sum_{i=1, i \neq j}^{I} \sum_{k=1}^{K}\left|\sqrt{\beta_{j k}^{i}} \boldsymbol{h}_{j k}^{i^{T}} \boldsymbol{a}_{i k}\right|^{2}}
$$

Assume that the maximum power of per base station is $P$. In order to optimize the transmission power, $\varphi$ is defined as the utilization rate of the transmission power and $\phi \in[0,1]$. Under the condition that $\operatorname{SINR}_{j k}$ of user $k$ in cell $j$ is greater than the desired threshold $\gamma_{j k}$, the objective of downlink Massive MIMO precoding should ensure to maximize the utilization rate of the transmission power and minimize the total power consumption. So the objective function is written as following:

$$
\begin{aligned}
& \min { }_{\varphi,\left\{\boldsymbol{a}_{j k}\right\}} \varphi P \\
& \text { st } \quad \sum_{k=1}^{K}\left|\boldsymbol{a}_{j k}\right|^{2} \leq \varphi P, \\
& \quad \operatorname{SINR} R_{j k} \geq \gamma_{j k}, \forall k=1 \ldots \ldots K
\end{aligned}
$$

Assume that $\mu_{j}$ is defined as Lagrange coefficients of the maximum power $P$, and $\frac{\lambda_{j k}}{N_{t}}$ is defined as Lagrange coefficient of SINR of user $k$ in cell $j$. Lagrange equation is expressed as following: 


$$
\begin{aligned}
& L\left(\phi, \boldsymbol{a}_{j k}, \frac{\lambda_{j k}}{N_{t}}, \mu_{j}\right)=P \phi+\mu_{j} \sum_{k}\left[\left|\boldsymbol{a}_{j k}\right|^{2}-\phi P\right]+\sum_{k} \frac{\lambda_{j k}}{N_{t}}\left[1+\left|\sum_{l=1, l \neq j}^{L} \sum_{k=1}^{K} \sqrt{\beta_{l k}^{j}} \boldsymbol{h}_{l k}^{j^{T}} \boldsymbol{a}_{j k}\right|^{2}\right. \\
& \left.+\sum_{i=1, i \neq j}^{I} \sum_{k=1}^{K}\left|\sqrt{\beta_{j k}^{i}} \boldsymbol{h}_{j k}^{i{ }^{T}} \boldsymbol{a}_{i k}\right|^{2}-\frac{1}{\gamma_{j k}}\left|\hat{\boldsymbol{H}}_{j k}^{j^{T}} \boldsymbol{a}_{j k}\right|^{2}\right]
\end{aligned}
$$

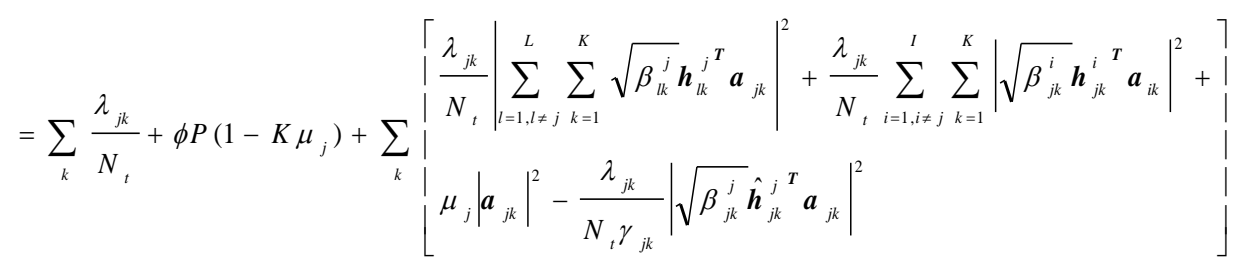

The channel information $\boldsymbol{h}_{j k}^{i}$ from $k$ UEs of cell $j$ to Base Station of cell $i$ and the precoding matrix $\boldsymbol{a}_{i k}$ need to be acquired in cell $i$. To reduce the complication of channel information feedback, we can consider SLNR criterion and utilize the channel reciprocity. The less power base station in cell $j$ leakage to other cells, the less interference users of cell $j$ sustain. So it can be assumed that the leakage power of the base station of cell $j$ is in proportion to the interference power of user in cell $j$. According to SLNR criterion, the leaking power of the base station in cell $j$ is $\sum_{i=1, i \neq j}^{I} \sum_{k=1}^{K}\left|\sqrt{\beta_{i k}^{j}} \boldsymbol{h}_{i k}^{j^{T}} \boldsymbol{a}_{j k}\right|^{2}$, and replaces the interference power $\sum_{i=1, i \neq j}^{I} \sum_{k=1}^{K}\left|\sqrt{\beta_{j k}^{i}} \boldsymbol{h}_{j k}^{{ }^{i}{ }^{T}} \boldsymbol{a}_{i k}\right|^{2}$ in (9). Thus (9) can be rewritten as following:

$$
\begin{aligned}
& L\left(\phi, \boldsymbol{a}_{j k}, \frac{\lambda_{j k}}{N_{t}}, \mu_{j}\right)=\sum_{k} \frac{\lambda_{j k}}{N_{t}}+\phi P\left(1-K \mu_{j}\right) \\
& +\sum_{k} \boldsymbol{a}_{j k}^{H}\left\lfloor\mu_{j} \boldsymbol{I}+\frac{\lambda_{j k}}{N_{t}}\left(\sum_{l=1, l \neq j}^{L} \sum_{k=1}^{K} \beta_{l k}^{j}\left(\boldsymbol{h}_{l k}^{j^{T}}\right)^{H} \boldsymbol{h}_{l k}^{j^{T}}+\sum_{i=1, i \neq j}^{I} \sum_{k=1}^{K} \beta_{i k}^{j}\left(\boldsymbol{h}_{i k}^{j^{T}}\right)^{H} \boldsymbol{h}_{i k}^{j^{T}}-\frac{1}{\gamma_{j k}} \beta_{j k}^{j}\left(\hat{\boldsymbol{h}}_{j k}^{j^{T}}\right)^{H} \hat{\boldsymbol{h}}_{j k}^{j^{j}}\right)\right\rceil \boldsymbol{a}_{j k}
\end{aligned}
$$

We define $\boldsymbol{\Pi}_{j k}=\mu_{j} \boldsymbol{I}+\frac{\lambda_{j k}}{N_{t}}\left(\sum_{l=1, l \neq j}^{L} \sum_{k=1}^{K} \beta_{l k}{ }_{l k}\left(\boldsymbol{h}_{l k}^{j^{T}}\right)^{H} \boldsymbol{h}_{l k}^{j^{T}}+\sum_{i=1, i \neq j}^{I} \sum_{k=1}^{K} \beta_{i k}^{j}\left(\boldsymbol{h}_{i k}^{j^{T}}\right)^{H} \boldsymbol{h}_{i k}^{j^{\boldsymbol{T}}}\right)$, so (10) can be simplified to (11):

$$
L\left(\varphi, \boldsymbol{a}_{j k}, \frac{\lambda_{j k}}{N_{t}}, \mu_{j}\right)=\sum_{k} \frac{\lambda_{j k}}{N_{t}}+\varphi P\left(1-K \mu_{j}\right)+\sum_{k} \boldsymbol{a}_{j k}^{H}\left(\boldsymbol{\Pi}_{j k}-\frac{\lambda_{j k}}{\gamma_{j k} N_{t}} \beta_{j k}^{j}\left(\hat{\boldsymbol{h}}_{j k}^{{ }^{T}}\right)^{H} \hat{\boldsymbol{h}}_{j k}^{j}\right) \boldsymbol{a}_{j k}
$$

Note that the KKT conditions, it should satisfy $h(x)=0$ and $a^{*} g(x)=0$ in the Lagrange equation $L(\mathrm{a}, \mathrm{b}, \mathrm{x})=f(\mathrm{x})+\mathrm{a} * \mathrm{~g}(\mathrm{x})+\mathrm{b} * \mathrm{~h}(\mathrm{x})$ containing $\mathrm{g}(\mathrm{x})$. To satisfy $\mathrm{a}^{*} \mathrm{~g}(\mathrm{x})=0$, it can be deduced $a=0$ or $g(\mathrm{x})=0$.

$$
\begin{aligned}
& \text { For } \sum_{k} \boldsymbol{a}_{j k}^{H}\left(\boldsymbol{\Pi}{ }_{j k}-\frac{\lambda_{j k}}{\gamma_{j k} N_{t}} \beta_{j k}^{j}\left(\hat{\boldsymbol{h}}_{j k}^{j}\right)^{T} \hat{\boldsymbol{h}}_{j k}^{{ }^{T}}\right) \boldsymbol{a}_{j k} \text {, that is } \boldsymbol{a}_{j k}^{H}=0 \text { or } \\
& \left(\boldsymbol{\Pi}_{j k}-\frac{\lambda_{j k}}{\gamma_{j k} N_{t}} \beta_{j k}^{j}\left(\hat{\boldsymbol{h}}_{j k}^{j}\right)^{T} \hat{\boldsymbol{h}}_{j k}^{{ }^{j}}{ }^{\boldsymbol{T}}\right) \boldsymbol{a}_{j k}=0 . \boldsymbol{a}_{j k}^{H} \text { is the precoding vector which can't be }
\end{aligned}
$$
zero, so we can deduce the formula as following: 


$$
\left(\boldsymbol{\Pi}_{j k}-\frac{\lambda_{j k}}{\gamma_{j k} N_{t}} \beta_{j k}^{j}\left(\hat{\boldsymbol{h}}_{j k}^{j}\right)^{H} \hat{\boldsymbol{h}}_{j k}^{j}\right) \boldsymbol{a}_{j k}=0
$$

Because $\Pi_{j k}$ is a non-singular matrix (i.e. $\left|\Pi_{j k}\right| \neq 0$ ) and optimal $\lambda_{j k}$ is strictly positive, we can deduce $\lambda_{j k}$ which satisfies $\left(\boldsymbol{\Pi}_{j k}-\frac{\lambda_{j k}}{\gamma_{j k} N_{t}} \beta_{j k}^{j}\left(\hat{\boldsymbol{h}}_{j k}^{j^{T}}\right)^{H} \hat{\boldsymbol{h}}_{j k}^{{ }^{T}}\right) \boldsymbol{a}_{j k}=0$ as following:

$$
\lambda_{j k}=\frac{N_{t} \gamma_{j k}}{\beta_{j k}^{j}\left(\hat{\boldsymbol{h}}_{j k}^{{ }^{T}}\right)^{H} \boldsymbol{\Pi}_{j k}{ }^{-1} \hat{\boldsymbol{h}}_{j k}^{{ }^{T}}}
$$

We substitute (13) into an equation (12), then rewrite the equation as following:

$$
\boldsymbol{\Pi}_{j k} \boldsymbol{a}_{j k}=\frac{\lambda_{j k}}{\gamma_{j k} N_{t}} \beta_{j k}^{j}\left(\hat{\boldsymbol{h}}_{j k}^{j}\right)^{H} \hat{\boldsymbol{h}}_{j k}^{{ }^{\boldsymbol{T}}} \boldsymbol{a}_{j k}
$$

And $\Pi_{j k}$ is a non-singular matrix, (14) can be written as following:

$$
\boldsymbol{a}_{j k}=\frac{\lambda_{j k}}{\gamma_{j k} N_{t}} \beta_{j k}^{j}\left(\hat{\boldsymbol{h}}_{j k}^{j}\right)^{H} \hat{\boldsymbol{h}}_{j k}^{j^{T}} \boldsymbol{\Pi} \boldsymbol{\Pi}_{j k}^{-1} \boldsymbol{a}_{j k}=\left(\frac{\lambda_{j k} \beta_{j k}^{j}}{\gamma_{j k} N_{t}} \hat{\boldsymbol{h}}_{j k}^{j^{T}} \boldsymbol{a}_{j k}\right) \boldsymbol{\Pi}_{j k}{ }^{-1}\left(\hat{\boldsymbol{h}}_{j k}^{j}\right)^{\boldsymbol{T}}=\psi \boldsymbol{\Pi}_{j k}{ }^{-1}\left(\hat{\boldsymbol{h}}_{j k}^{{ }^{j}}\right)^{H}
$$

$\frac{\lambda_{j k} \beta_{j k}^{j}}{\gamma_{j k} N_{t}} \hat{\boldsymbol{h}}_{j k}^{{ }^{T}}{ }^{T} \boldsymbol{a}_{j k}$ is the dimension with $1 \times 1$ and $\frac{\lambda_{j k} \beta_{j k}^{j}}{\gamma_{j k} N_{t}} \hat{\boldsymbol{h}}_{j k}^{{ }^{T} \boldsymbol{T}} \boldsymbol{a}_{j k}=\psi$. Where $\psi$ is a scalar to be determined. According to (15), the optimal vector $\boldsymbol{a}_{j k}$ can be expressed as following:

$$
\boldsymbol{a}_{j k}=\psi \boldsymbol{\Pi}_{j k}^{-1}\left(\hat{\boldsymbol{h}}_{j k}{ }^{\boldsymbol{T}}\right)^{H}
$$

\section{Numerical Results}

The proposed Max-SINR algorithm is evaluated with the link-level simulation platform. In the simulations, it is compared with matched-filter (MF) algorithm [2]. The downlink precoding matrix of the base station in the cell $j$ is calculated by $a_{j k}=\hat{\boldsymbol{h}}_{j k}^{{ }^{T}} /\left\|\hat{\boldsymbol{h}}_{j k}^{{ }^{T}}\right\|$ according to MF algorithm. The proposed algorithm will calculate the downlink precoding matrix according (16).

In the simulation, the impact of pilot contamination will be evaluated. There are three cells and each Base Station configures 100 antennas spacing $0.5 \lambda$ and serves 10 users with single antenna. The transmission power of base station is $20 \mathrm{~dB}$ and the transmission power of each user is $1 \mathrm{~dB}$. The modulation scheme is 16QAM and channel coding is $1 / 2$ convolutional code.

Cell 2 reuses the same pilot sequences with cell 1 , and cell 3 uses orthogonal pilot sequences. When the large-scale fading coefficients of cell 2 became smaller, it means users of cell 1 sustain more serious pilot contamination. When the large-scale fading coefficients of cell 3 became smaller, it means users of cell 1 sustain more serious inference from other cell.

In Figure 3 (a), we assume that the large fading coefficients are set to $\beta_{1 k}^{1}=0.8$, $\beta_{2 k}^{1}=0.1$ and $\beta_{3 k}^{1}=0.1$. Under this condition the pilot contamination from cell 2 to cell 1 is light. We evaluate the spectrum efficiency of MF and the proposed algorithm from 20 antennas to 100 antennas. It can be shown that the proposed algorithm outperform $\mathrm{MF}$ algorithm. The more antennas the base station has, the bigger the gap of performance is. 
When the number of antenna are 20, the spectrum efficiency of the proposed algorithm is 1.12 times higher than MF algorithm; when the number of antenna are 100, the spectrum efficiency of the proposed algorithm is 1.3 times higher than MF algorithm

Assume that the large fading coefficients are set to $\beta_{1 k}^{1}=0.1$, $\beta_{2 k}^{1}=0.1$ and $\beta_{3 k}^{1}=0.1$ in the simulation of Figure $3(\mathrm{~b})$. Under this condition the pilot contamination from cell 2 to cell 1 is severe. It is shown that MF algorithm can't get obvious performance gains with the number of antenna increasing. The performance gap of two algorithms is bigger than the condition with light pilot contamination. When the number of antenna are 20, the spectrum efficiency of the proposed algorithm is 1.24 times higher than MF algorithm, when the number of antenna are 100, the spectrum efficiency of the proposed algorithm is 1.9 times higher than MF algorithm.

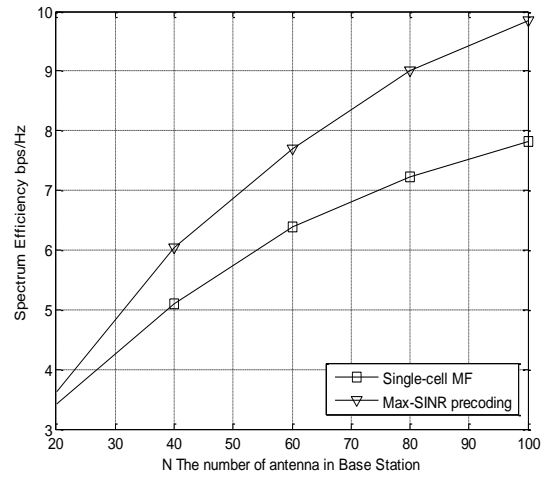

(a)

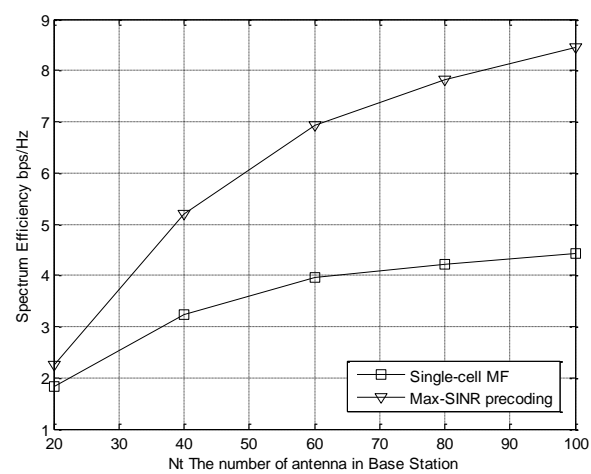

(b)

Figure 3. Simulation Results of Massive MIMO Precoding (a) Light Pilot Contamination; (b) Severe Pilot Contamination

\section{Conclusion}

Numerical results proved that Max-SINR precoding algorithm outperform than the traditional Massive MIMO precoding --- MF algorithm. For example, the spectrum efficiency of the proposed algorithm is 1.9 times higher than MF algorithm when the pilot contamination is very severe. Moreover the performance gap of two algorithms is more obvious with the number of antenna increasing. So the proposed algorithm can effectively mitigate the performance degrade caused by pilot contamination and achieve high throughput with maximizing the gains of transmitting power.

We assume that Massive MIMO channel is completely independent in this paper, but actual Massive MIMO channel is rather complicated. In the future study, we will investigate the proposed algorithm in different channel environments.

\section{Acknowledgments}

This paper is sponsored by National Natural Science Foundation of China (61102047) and the Science Research Project of Shaanxi Provincial Department of Education (11JK1016).

\section{References}

[1] T. L. Marzetta, "Non cooperative cellular wireless with unlimited numbers of base station antennas", IEEE Trans. Wireless Commun, vol. 9, no. 11, (2010).

[2] F. Rusek, D. Persson, B. K. Lau, E. G. Larsson et al., "Scaling up MIMO: Opportunities and challenges with very large arrays", IEEE Signal Process. Mag, vol. 30, no. 1, (2012). 
[3] C. Lee and C.-B. Chae, "Network Massive MIMO for Cell-Boundary Users: From a Precoding Normalization Perspective", GC'12 Workshop: International Workshop on Cloud Base-Station and Large-Scale Cooperative Communications, (2012), pp. 233-237.

[4] J. Hoydis and S. T. Brink, "Massive MIMO in the UL/DL of Cellular Networks", How Many Antennas Do We Need?, IEEE Journal on Selected Areas in Communications, vol. 31, no. 2, (2013).

[5] W. Yang, G. Durisi and E. Riegler, "On the Capacity of Large-MIMO Block-Fading Channels", IEEE Journal On Selected Areas In Communications, vol. 31, no. 2, (2013).

[6] H. Q. Ngo, E. G. Larsson and T. L. Marzetta, "Energy and Spectral Efficiency of Very Large Multiuser MIMO Systems", IEEE Transactions on Communications, vol. 61, no. 4, (2013.

[7] X. Gao, O. Edfors and F. Rusek, "Linear pre-coding performance in measured very-large MIMO channels", IEEE Vehicular Technology Conference (VTC Fall), (2011), pp. 1-5.

[8] H. Yang and T. L. Marzetta, "Performance of Conjugate and Zero-Forcing Beamforming in Large-Scale Antenna Systems", IEEE Journal on Selected Areas in Communications, vol. 31, no. 2, (2011).

[9] O. N. Alrabadi, E. Tsakalaki and H. Huang, "Beamforming via Large and Dense Antenna Arrays Above a Clutter", IEEE Journal On Selected Areas In Communications, vol. 31, no. 2, (2013).

[10] C. Guthy, W. Utschick and M. L. Honig, "Large System Analysis of the Successive Encoding Successive Allocation Method for the MIMO BC", 2010 International ITG Workshop on Smart Antennas (WSA), (2010), pp. 226-231.

[11] C. Artigue and P. Loubaton, "On the Precoder Design of Flat Fading MIMO Systems Equipped With MMSE Receivers", A Large-System Approach. IEEE Transactions On Information Theory, vol. 57, no. 7, (2011).

[12] J. Jose, A. Ashikhmin and T. L. Marzetta, "Pilot Contamination and Precoding in Multi-Cell TDD Systems", IEEE Transactions On Wireless Communications, vol. 10, no. 8, (2011).

[13] K. Appaiah, A. Ashikhmin and T. L. Marzetta, "Pilot Contamination Reduction in Multi-user TDD Systems", IEEE International Conference on Communications (ICC), (2010), pp.1-5.

[14] A. Ashikhmin and T. Marzetta, "Pilot Contamination Precoding in Multi-Cell Large Scale Antenna System", 2012 IEEE International Symposium on Information Theory Proceedings, (2012), pp. 1137-1141.

[15] S. Payami and F. Tufvesson, "Channel Measurements and Analysis for Very Large Array Systems at 2.6 GHz", 6th European Conference on Antennas and Propagation (EUCAP), (2012), pp. 433-437.

[16] Q. Zhou and X. Ma, "Element-Based Lattice Reduction Algorithms for Large MIMO Detection”, IEEE Journal on Selected Areas in Communications, vol. 31, no. 2, (2013).

[17] A. O"zgu"r and O. Le've^que, "Spatial Degrees of Freedom of Large Distributed MIMO Systems and Wireless Ad Hoc Networks", IEEE Journal On Selected Areas In Communications, vol. 31, no. 2, (2013).

[18] S. Xi and M. D. Zoltowski, "Iterative SINR-MAX Cooperative Beamforming In Multiuser MIMO System”, IEEE Sarnoff Symposium, (2008), pp.3237-3240.

[19] Z. Xiang and M. Tao, "Coordinated Multicast Beamforming in Multicell Networks", IEEE Transactions On Wireless Communications, vol. 12, no. 1, (2013).

[20] S. Xi and M. D. Zoltowski, "SINR-MAX Cooperative Beamforming For Multi-user MIMO-OFDM Systems", ICASSP, (2008), pp. 3237-3240.

[21] S. He, Y. Huang and H. Wang, "Coordinated Multi-cell Beamforming Scheme Using Uplink-Downlink Max-Min SINR Duality”, Globecom 2012-Signal Processing for Communications Symposium, (2012), pp. 3930-3934.

[22] D. W. H. Cai and T. Q. S. Quek, "Max-Min SINR Coordinated Multipoint Downlink Transmission-Duality and Algorithms", IEEE Transactions On Signal Processing, vol. 60, no, 10, (2012).

[23] S. Mukherjee and P. Alto, "Downlink SINR distribution in a Heterogeneous Cellular Wireless Network with max-SINR Connectivity", Forty-Ninth Annual Allerton Conference Allerton House, (2011), pp.1649-1656.

[24] C. Wilson and V. Veeravalli, "A Convergent Version of the Max SINR Algorithm for the MIMO Interference Channel”, IEEE Transactions On Wireless Communications, vol. 12, no. 6, (2013).

[25] F. Chen and W. Su, "Joint Power Optimization for Multi-Source Multi-Destination Relay Networks", IEEE Transactions On Signal Processing, vol. 59, no. 5, (2011). 


\section{Authors}

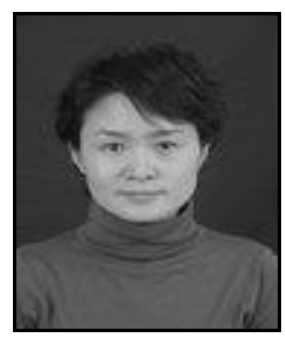

Jiang Jing, she received M. Sc. from the XiDian University in 2005 and a Ph.D. in Information and Communication Engineering from North Western Polytechnic University, China in 2009. She had been a researcher and a project manager from 2006 to 2012 at ZTE Corporation in China, and currently as an associate professor of School of Communication and Information Engineering in Xi'an University of Posts \& Telecommunications, China. Her research interests include wireless communication theory and MIMO system design. She has been a Member of the IEEE, 3GPP.

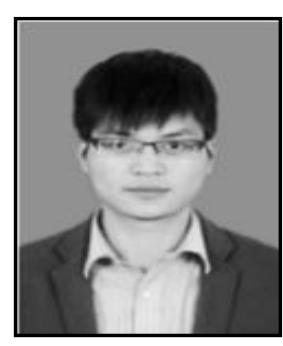

Xu Zheng, he was born in Zhejiang Province, China, in 1990. He received the B.E. degree from Chang'an College of XiDian University, Xi'an, in 2012. He is currently studying as a M.E. in School of Communication and Information Engineering in Xi'an University of Posts \& Telecommunications, China. His research interests include CoMP, Massive MIMO. 